\section{Unexpected identification of bilateral masses in an asymptomatic heavy smoker}

A 47-year-old male heavy smoker who was an ex-intravenous drugs user presented with gallstones; a preoperatived chest radiograph showed hazy opacity in the right lower lobe. Chest CT revealed in the lower posterior lobes two almost symmetrical areas of consolidation with fat attenuation values (-50 Hounsfield units, figure 1). A broncholaveolar lavage did not provide pathological findings. A transthoracic core needle biopsy showed the pattern of chronic exogenous lipoid pneumonia, with numerous lipid-laden macrophages (figure 2). Upon further investigation, the patient revealed that he had been taking $200 \mathrm{ml}$ of mineral oil daily for 5 years to treat chronic constipation related to methadone treatment.

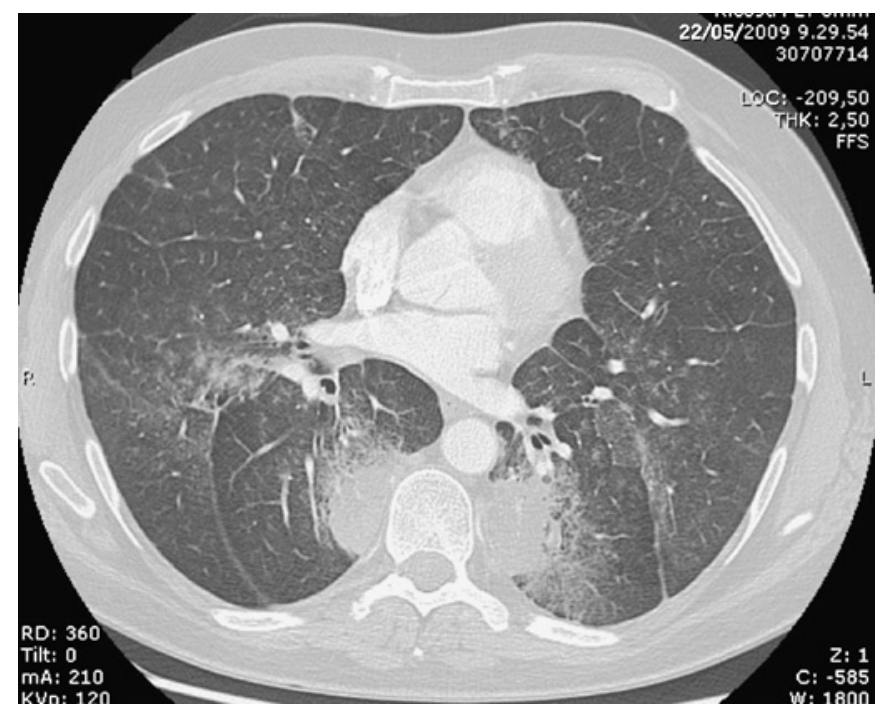

Figure 1 CT scan of the chest with the lung window showing two almost symmetrical areas of consolidation in the lower posterior lobes; surrounding parenchyma was characterised by ground-glass opacities with interlobular septal thickening, suggesting a 'crazy-paving' pattern.

\section{Elisa Garlassi, ${ }^{1}$ Giulio Rossi, ${ }^{2}$ Andrea Bedini, ${ }^{2}$ Luca Richeldi ${ }^{1}$ \\ ${ }^{1}$ University of Modena \& Reggio Emilia, Modena, Italy; ${ }^{2}$ Policlinico Hospital of Modena, Modena, Italy}

Correspondence to Luca Richeldi, Centre for Rare Lung Disease, Policlinico Hospital, University of Modena \& Reggio Emilia, Modena 41124, Italy; luca.richeldi@unimore.it

\section{Competing interests None.}

Patient consent Obtained.

Provenance and peer review Not commissioned; externally peer reviewed.

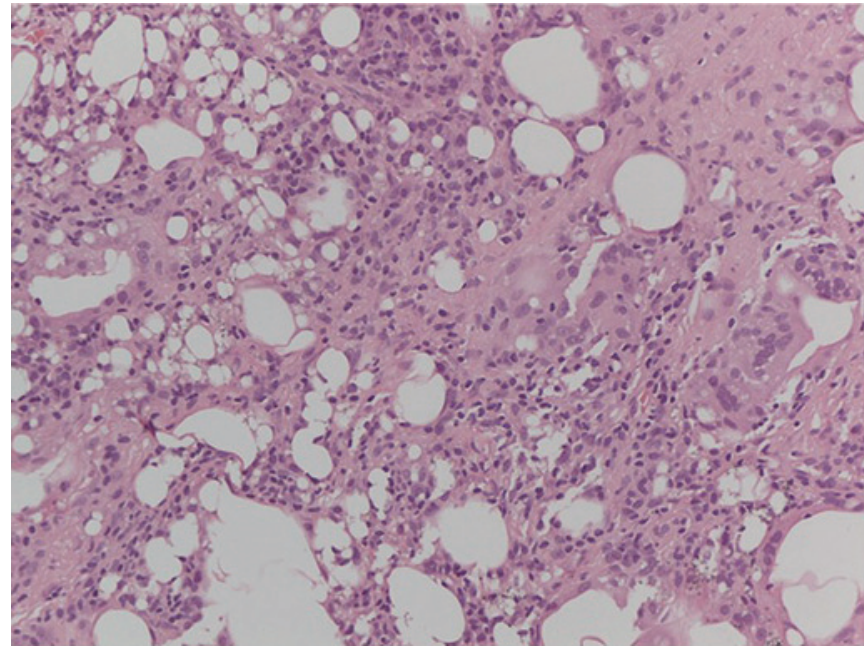

Figure 2 Transthoracic biopsy (performed using an 18-gauge needle as the guide and a 20-gauge gun to obtain the core) showing the round-tooval vacuolar spaces representing lipid vacuoles that are washed out during tissue processing (H\&E stain, $\times 400$ magnification).

\section{Learning points}

- Lipoid pneumonia can be totally asymptomatic even in heavy smokers and even when lipoid masses are considerable in size. ${ }^{1}$

- Although the diagnosis of lipoid pneumonia is uncommon, it still needs to be considered in adults without anatomical abnormalities predisposing to aspiration. ${ }^{2}$

- The finding of a lung mass surrounded by a crazy-paving pattern on chest CT with density values indicating the presence of fat tissue (Hounsfield units within the -60 to +10 range) should trigger minimally invasive bioptic techniques that may be diagnostic, avoiding surgical approaches.

- Triggers for exogenous lipoid pneumonia are usually not recognised as risk factors by patients and as such are not reported.

Accepted 28 December 2009

Published Online First 29 July 2010

Thorax 2010;65:846. doi:10.1136/thx.2009.130500

\section{REFERENCES}

1. Spickard A 3rd, Hirschmann JV. Exogenous lipoid pneumonia. Arch Intern Med 1994:154:686-92.

2. Gondouin A, Manzoni P, Ranfaing E, et al. Exogenous lipid pneumonia: a retrospective multicentre study of 44 cases in France. Eur Respir $\mathrm{J}$ 1996; 9:1463-9. 